\title{
Excited isovector mesons using the stochastic LapH method
}

\author{
Colin Morningstar ${ }^{* a}$, Brendan Fahy ${ }^{a, b}$, You-Cyuan Jhang ${ }^{a}$, Keisuke J. Juge ${ }^{c}$, \\ David Lenkner $^{d}$, and Chik Him Wong ${ }^{e}$ \\ ${ }^{a}$ Dept. of Physics, Carnegie Mellon University, Pittsburgh, PA 15213, USA \\ ${ }^{b}$ High Energy Accelerator Research Organization (KEK), Ibaraki 305-0801, Japan \\ ${ }^{c}$ Dept. of Physics, University of the Pacific, Stockton, CA 95211, USA \\ ${ }^{d}$ Data Science Automation, 375 Valley Brook Road, Pittsburgh, PA 15317, USA \\ ${ }^{e}$ Department of Physics, University of Wuppertal, Gaussstrasse 20, D-42119, Germany
}

\begin{abstract}
The spectrum of excited isovector mesons is studied using a $32^{3} \times 256$ anisotropic lattice with $u, d$ quark masses set to give a pion mass near $240 \mathrm{MeV}$. Results in the bosonic isovector nonstrange symmetry channels of zero total momentum are presented using correlation matrices of unprecedented size. In addition to spatially-extended single-meson operators, large numbers of two-meson operators are used, involving a wide variety of light isovector, isoscalar, and strange meson operators of varying relative momenta. All needed Wick contractions are efficiently evaluated using a stochastic method of treating the low-lying modes of quark propagation that exploits Laplacian Heaviside quark-field smearing. Level identification is discussed.
\end{abstract}

The 32nd International Symposium on Lattice Field Theory,

23-28 June, 2014

Columbia University New York, NY

${ }^{*}$ Speaker. 


\section{Introduction}

In a series of papers[1, 2, 3, 4, 5, 6], we have been striving to compute the finite-volume stationary-state energies of QCD using Markov-chain Monte Carlo integration of the QCD path integrals formulated on a space-time lattice. In this talk, our progress made during the past year is described. Last year, we presented our first results in the zero-momentum bosonic $I=1, S=0, T_{1 u}^{+}$ symmetry sector of QCD on small $24^{3} \times 128$ lattices with an unphysically heavy pion mass around $390 \mathrm{MeV}$. Here, we report on results obtained on a $32^{3} \times 256$ anisotropic lattice for which the pion mass is around $240 \mathrm{MeV}$. All needed Wick contractions are efficiently evaluated using a stochastic method of treating the low-lying modes of quark propagation that exploits Laplacian Heaviside quark-field smearing. Given the large number of levels extracted, level identification becomes a key issue.

\section{Operators, configurations, and analysis}

The stationary-state energies in a particular symmetry sector can be extracted from an $N \times N$ Hermitian correlation matrix $\mathscr{C}_{i j}(t)=\left\langle 0\left|O_{i}\left(t+t_{0}\right) \bar{O}_{j}\left(t_{0}\right)\right| 0\right\rangle$, where the $N$ operators $\bar{O}_{j}$ act on the vacuum to create the states of interest at source time $t_{0}$ and are accompanied by conjugate operators $O_{i}$ that can annihilate these states at a later time $t+t_{0}$. Estimates of $\mathscr{C}_{i j}(t)$ are obtained with the Monte Carlo method using the stochastic LapH method[5] which allows all needed quark-line diagrams to be computed.

All of our single-hadron operators are assemblages of basic building blocks which are gaugecovariantly-displaced, LapH-smeared quark fields, as described in Refs. [1, 5, 6]. Each of our single-hadron operators creates and annihilates a definite momentum. Group-theoretical projections are used to construct operators that transform according to the irreducible representations of the space group $O_{h}^{1}$, plus $G$-parity, when appropriate. In order to build up the necessary orbital and radial structures expected in the hadron excitations, we use a variety of spatially-extended configurations. For practical reasons, we restrict our attention to certain classes of momentum directions for the single hadron operators: on axis $\pm \widehat{\boldsymbol{x}}, \pm \widehat{\boldsymbol{y}}, \pm \widehat{\boldsymbol{z}}$, planar diagonal $\pm \widehat{\boldsymbol{x}} \pm \widehat{\boldsymbol{y}}, \pm \widehat{\boldsymbol{x}} \pm \widehat{\boldsymbol{z}}, \pm \widehat{\boldsymbol{y}} \pm \widehat{\boldsymbol{z}}$, and cubic diagonal $\pm \widehat{\boldsymbol{x}} \pm \widehat{\boldsymbol{y}} \pm \widehat{\boldsymbol{z}}$. However, some special momentum directions, such as $\pm 2 \widehat{\boldsymbol{x}} \pm \widehat{\boldsymbol{y}}$, are used. We construct our two-hadron operators as superpositions of single-hadron operators of definite momenta. Again, group-theoretical projections are employed to produce two-hadron operators that transform irreducibly under the symmetry operations of our system. This approach is efficient for creating large numbers of two-hadron operators, and generalizes to three or more hadrons.

In finite volume, all energies are discrete so that each correlator matrix element has a spectral representation of the form

$$
\mathscr{C}_{i j}(t)=\sum_{n} Z_{i}^{(n)} Z_{j}^{(n) *} e^{-E_{n} t}, \quad Z_{j}^{(n)}=\left\langle 0\left|O_{j}\right| n\right\rangle,
$$

assuming temporal wrap-around (thermal) effects are negligible. We extract energies from our correlation matrices using a "single rotation" or "fixed coefficient" method. Starting with a raw correlation matrix $\mathscr{C}(t)$, we first try to remove the effects of differing normalizations by forming the matrix $C_{i j}(t)=\mathscr{C}_{i j}(t)\left(\mathscr{C}_{i i}\left(\tau_{N}\right) \mathscr{C}_{j j}\left(\tau_{N}\right)\right)^{-1 / 2}$, taking $\tau_{N}$ at a very early time, such as $\tau_{N}=3$. 
We ensure that $C$ is positive definite and has a reasonable condition number. Standard projection methods can be used to remove problematic modes. We then solve the generalized eigenvector problem $A x=\lambda B x$ with $A=C\left(\tau_{D}\right)$ and $B=C\left(\tau_{0}\right)$ for particular choices of times $\tau_{0}$ and $\tau_{D}$ (see below). The eigenvectors obtained are used to "rotate" the correlator $C(t)$ into a correlator $G(t)$ for which $G\left(\tau_{0}\right)=1$, the identity matrix, and $G\left(\tau_{D}\right)$ is diagonal. At other times, $G(t)$ need not be diagonal. However, with judicious choices of $\tau_{0}$ and $\tau_{D}$, one finds that the off-diagonal elements of $G(t)$ remain zero within statistical precision for $t>\tau_{D}$. The rotated correlator is given by

$$
G(t)=U^{\dagger} C\left(\tau_{0}\right)^{-1 / 2} C(t) C\left(\tau_{0}\right)^{-1 / 2} U,
$$

where the columns of $U$ are the orthonormalized eigenvectors of $C\left(\tau_{0}\right)^{-1 / 2} C\left(\tau_{D}\right) C\left(\tau_{0}\right)^{-1 / 2}$. Rotated effective masses can then be defined by

$$
m_{G}^{(n)}(t)=\frac{1}{\Delta t} \ln \left(\frac{G_{n n}(t)}{G_{n n}(t+\Delta t)}\right)
$$

which tend to the lowest-lying $N$ stationary-state energies produced by the $N$ operators. Correlated$\chi^{2}$ fits to the estimates of $G_{n n}(t)$ using the forms

$$
A_{n} e^{-E_{n} t}\left(1+B_{n} e^{-\Delta_{n}^{2} t}\right)+A_{n} e^{-E_{n}(T-t)}\left(1+B_{n} e^{-\Delta_{n}^{2}(T-t)}\right),
$$

where $T$ is the temporal extent of the lattice, yield the energies $E_{n}$ and the overlaps $A_{n}$ to the rotated operators for each $n$. Using the rotation coefficients, one can then easily obtain the overlaps $Z_{j}^{(n)}=C\left(\tau_{0}\right)_{j k}^{1 / 2} U_{k n} A_{n}$ (no summation over $n$ ) corresponding to the rows and columns of the correlation matrix $C(t)$.

We are currently focusing on three Monte Carlo ensembles: (A) a set of 412 gauge-field configurations on a large $32^{3} \times 256$ anisotropic lattice with a pion mass $m_{\pi} \sim 240 \mathrm{MeV}$, (B) an ensemble of 551 configurations on an $24^{3} \times 128$ anisotropic lattice with a pion mass $m_{\pi} \sim 390 \mathrm{MeV}$, and (C) an ensemble of 584 configurations on an $24^{3} \times 128$ anisotropic lattice with a pion mass $m_{\pi} \sim 240 \mathrm{MeV}$. We refer to these ensembles as the $\left(32^{3} \mid 240\right),\left(24^{3} \mid 390\right)$, and $\left(24^{3} \mid 240\right)$ ensembles, respectively. These ensembles were generated using the Rational Hybrid Monte Carlo (RHMC) algorithm[9]. In each ensemble, successive configurations are separated by 20 RHMC trajectories to minimize autocorrelations. An improved anisotropic clover fermion action and an improved gauge field action are used[10]. In these ensembles, $\beta=1.5$ and the $s$ quark mass parameter is set to $m_{s}=-0.0743$ in order to reproduce a specific combination of hadron masses[10]. In the $\left(24^{3} \mid 390\right)$ ensemble, the light quark mass parameters are set to $m_{u}=m_{d}=-0.0840$ so that the pion mass is around $390 \mathrm{MeV}$ if one sets the scale using the $\Omega$ baryon mass. In the $\left(32^{3} \mid 240\right)$ and $\left(24^{3} \mid 240\right)$ ensembles, $m_{u}=m_{d}=-0.0860$ are used, resulting in a pion mass around $240 \mathrm{MeV}$. The spatial grid size is $a_{s} \sim 0.12 \mathrm{fm}$, whereas the temporal spacing is $a_{t} \sim 0.035 \mathrm{fm}$.

In our operators, a stout-link staple weight $\xi=0.10$ is used with $n_{\xi}=10$ iterations. For the cutoff in the LapH smearing, we use $\sigma_{s}^{2}=0.33$, which translates into the number $N_{v}$ of LapH eigenvectors retained being $N_{v}=112$ for the $24^{3}$ lattices and $N_{v}=264$ for the $32^{3}$ lattice. We use $Z_{4}$ noise in all of our stochastic estimates of quark propagation. Our variance reduction procedure is described in Ref. [5]. On the $24^{3}$ lattices, we use 4 widely-separated source times $t_{0}$, and 8 are used on the $32^{3}$ lattice. 

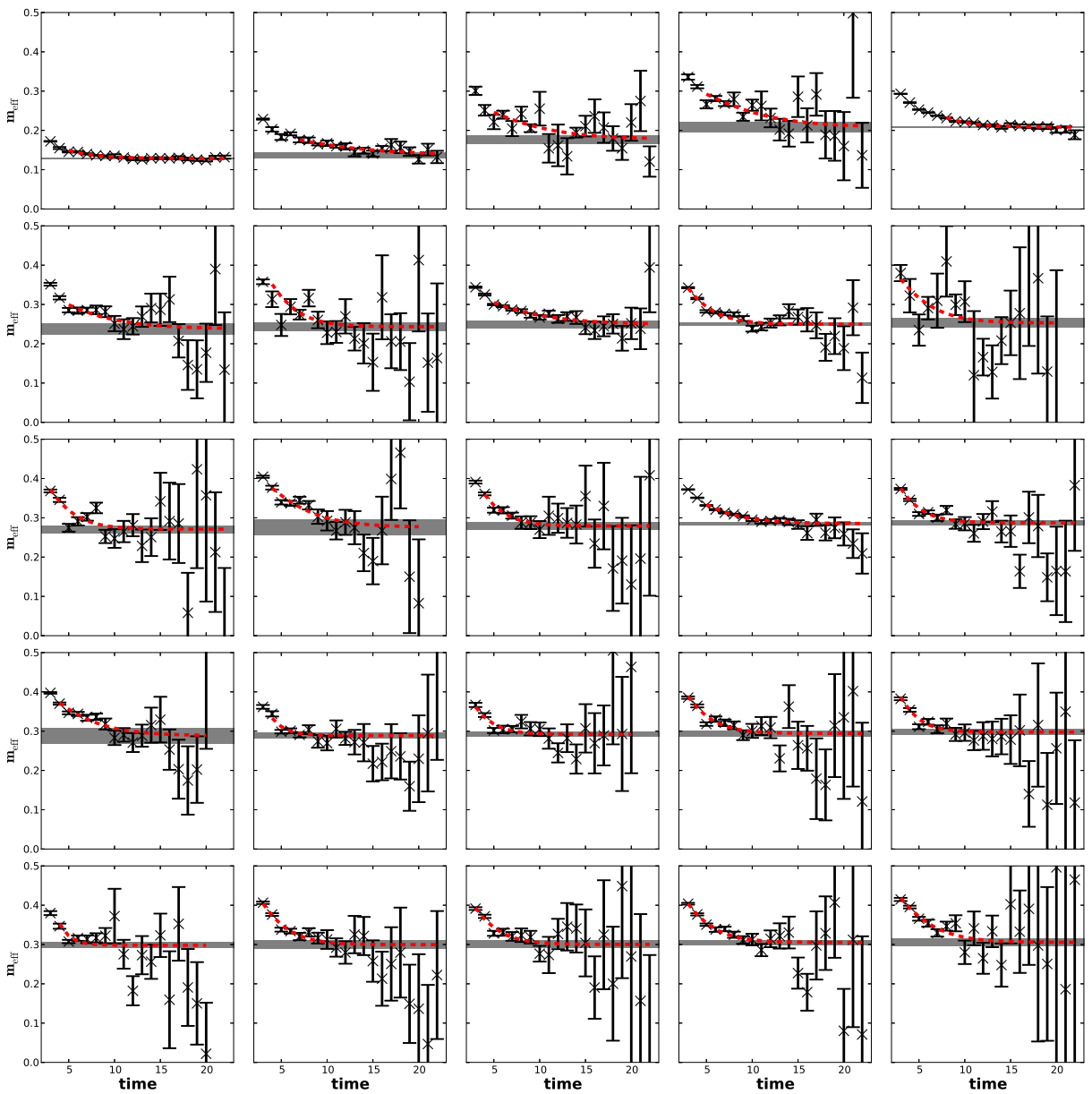

Figure 1: Rotated effective masses $m_{G}^{(n)}(t)$ (see Eq. (2.3)) for the 25 lowest-lying energy levels in the zeromomentum bosonic $I=1, S=0, T_{1 u}^{+}$channel for the $\left(32^{3} \mid 240\right)$ ensemble using 14 single-meson operators, 23 isovector+isovector operators, 31 light-isoscalar+isovector operators, $31 \bar{s} s$-isoscalar+isovector operators, and 9 kaon+antikaon operators. Dashed lines indicate energy extractions from correlated- $\chi^{2}$ fits. Gray bands show the best fit values of the energies, whose standard deviations are indicated by the width of each band.

\section{Energies in the $T_{1 u}^{+}$channel}

We focus here on the resonance-rich $I=1, S=0, T_{1 u}^{+}$channel of total zero momentum. This channel has odd parity, even $G$-parity, and contains the spin-1 and spin-3 mesons. Low statistics runs on smaller lattices led us to include 14 particular single-meson (quark-antiquark) operators. We took special care to include operators that could produce the spin-3 $\rho_{3}(1690)$ state, in addition to the other spin-1 states. Low statistics runs also gave us the masses of the lowest-lying mesons, such as the $\pi, \eta, K$, and so on. Given these known mesons, we used software written in MAPLE to find all possible two-meson states in our cubic box in this $T_{1 u}^{+}$symmetry channel, assuming no energy shifts from interactions or the finite volume. We used these so-called "expected two-meson levels" to guide our choice of two-meson operators to include. We included 23 isovector-isovector meson operators, 31 operators that combine an isovector with a light isoscalar (using only $u, d$ 

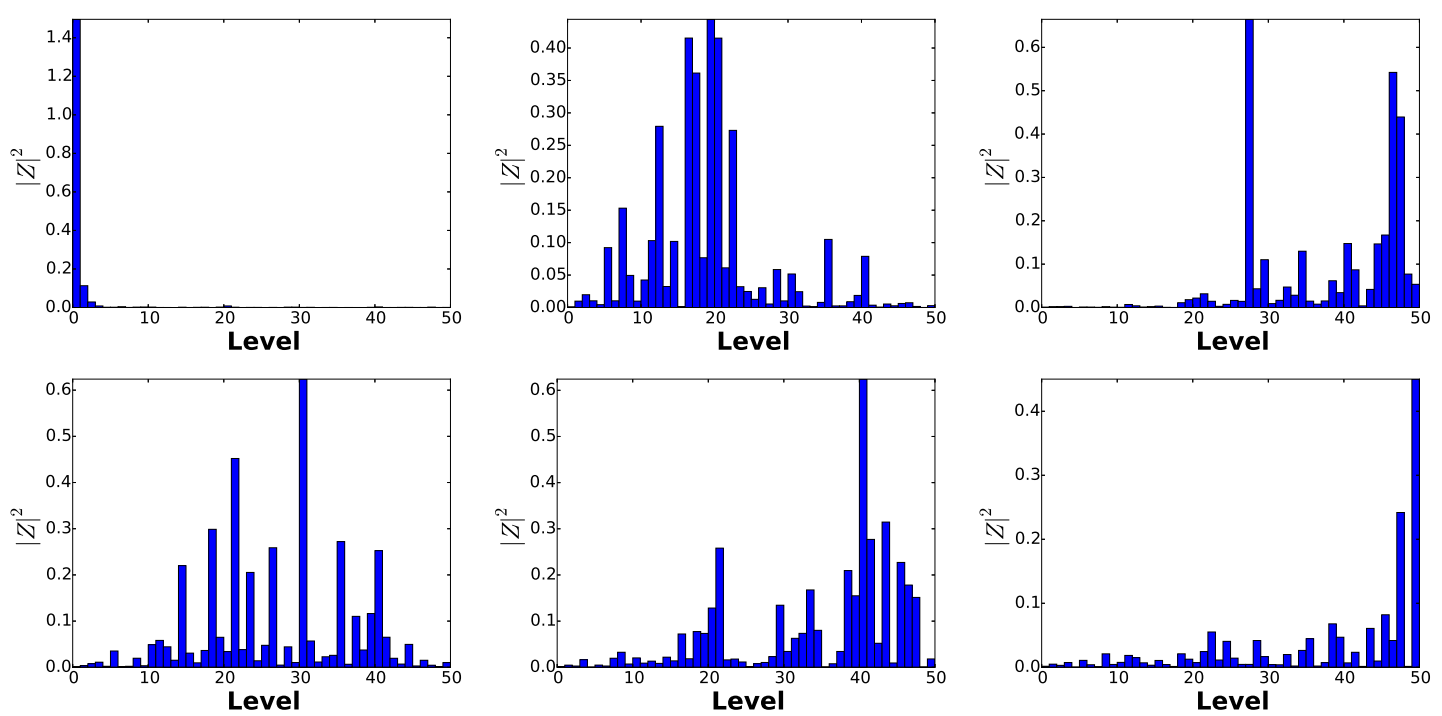

Figure 2: Overlaps $\left|\widetilde{Z}_{j}^{(n)}\right|^{2}$ of "optimized" single-hadron operator $\widetilde{O}_{j}$ against the eigenstates labelled by $n$. The overall normalization is arbitrary in each plot.

quarks), 31 operators that combine an isovector with an $\bar{s} s$ isoscalar meson, and 9 kaon-antikaon operators.

We obtained results for the lowest 50 energy levels using the $\left(32^{3} \mid 240\right)$ ensemble from our $108 \times 108$ correlation matrix. The rotated effective masses $m_{G}^{(n)}(t)$ (see Eq. (2.3)) using $\tau_{0}=5$ and $\tau_{D}=8$ are shown for the first 25 levels in Fig. 1. The results shown here are not finalized yet. We are still varying the fitting ranges to improve the $\chi^{2}$, as needed in some instances. We are investigating the effects of adding more operators, and we are even still verifying our analysis/fitting software. However, these figures do demonstrate that the extraction of a large number of energy levels is indeed possible, and the plots indicate the level of precision that can be attained with our stochastic LapH method. Keep in mind that we have not included any three-meson operators in our correlation matrix.

With such a large number of energies extracted, level identification becomes a key issue. QCD is a complicated interacting quantum field theory, so characterizing its stationary states in finite volume is not likely to be done in a simple way. Level identification must be inferred from the $Z$ overlaps of our probe operators, analogous to deducing resonance properties from scattering cross sections in experiments. Judiciously chosen probe operators, constructed from smeared fields, should excite the low-lying states of interest, with hopefully little coupling to unwanted higherlying states, and help with classifying the levels extracted. Small- $a$ classical expansions can help to characterize the probe operators, and hence, the states they produce.

We particularly wish to identify the finite-volume stationary-state levels expected to evolve into the single-meson resonances corresponding to quark-antiquark excitations in infinite volume. To accomplish this, we utilize "optimized" single-hadron operators as our probes. We first restrict our attention to the $14 \times 14$ correlator matrix involving only the 14 chosen single-hadron operators. We then perform an optimization rotation to produce so-called "optimized" single-hadron ( $\mathrm{SH}$ ) 
Tlup

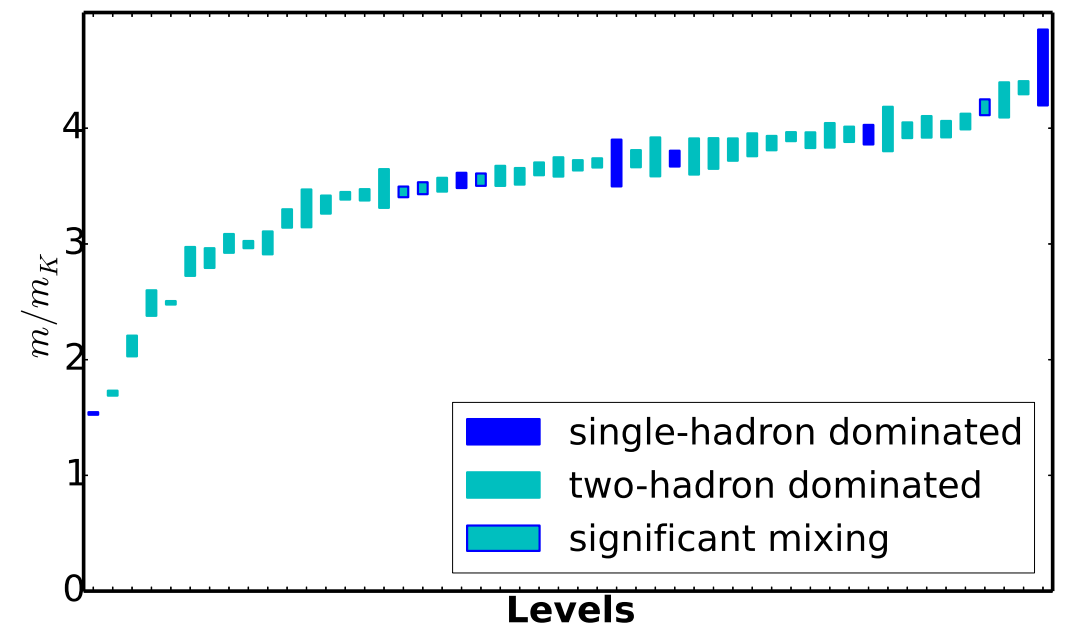

Figure 3: Energies $m$ as ratios of the kaon mass $m_{K}$ for the first fifty states excited by our single- and two-hadron operators in the $T_{1 u}^{+}$channel. For each optimized single-hadron operator, the level of maximum overlap is indicated by a solid blue box, and levels with overlaps greater than $75 \%$ of the largest are indicated by a dark blue outline.

operators $\widetilde{O}_{j}$, which are linear combinations of the 14 original operators, determined in a manner analogous to Eq. (2.2). We order these SH-optimized operators according to their effective mass plateau values, then evaluate the overlaps $\widetilde{Z}_{j}^{(n)}$ for these SH-optimized operators using our analysis of the full $108 \times 108$ correlator matrix. The results are shown in Fig. 2 .

Our energies in the $T_{1 u}^{+}$channel are summarized by the "staircase" plot in Fig. 3. For each SH optimized operator, the level with the largest overlap is identified on this plot using a solid blue box. Other levels with significant overlaps with the SH optimized operator are indicated by boxes with a dark blue outline. The remaining cyan boxes are levels with overlaps dominated by two-meson operators. The energies of the levels with solid blue boxes are collected and shown in Fig. 4, which compares these energies to experiment. The finite-volume energies should agree with experiment only within the widths of the infinite-volume resonances. We believe we have extracted all meson resonances that are quark-antiquark excitations. One observes more levels in experiment, although the experimental observations are controversial in some cases. Keep in mind that resonances that are not quark-antiquark excitations, such as so-called molecular states, would not be identified by our SH optimized operator overlaps. Again, we mention that three and four meson states are not taken into account at all.

\section{Conclusion}

In this talk, our progress made during the past year in computing the finite-volume stationarystate energies of QCD was described. Our results in the zero-momentum bosonic $I=1, S=0, T_{1 u}^{+}$ symmetry sector of QCD for the $\left(32^{3} \mid 240\right)$ ensemble using a correlation matrix of 108 operators were presented. All needed Wick contractions were efficiently evaluated using the stochastic LapH method. Issues related to level identification were discussed. 


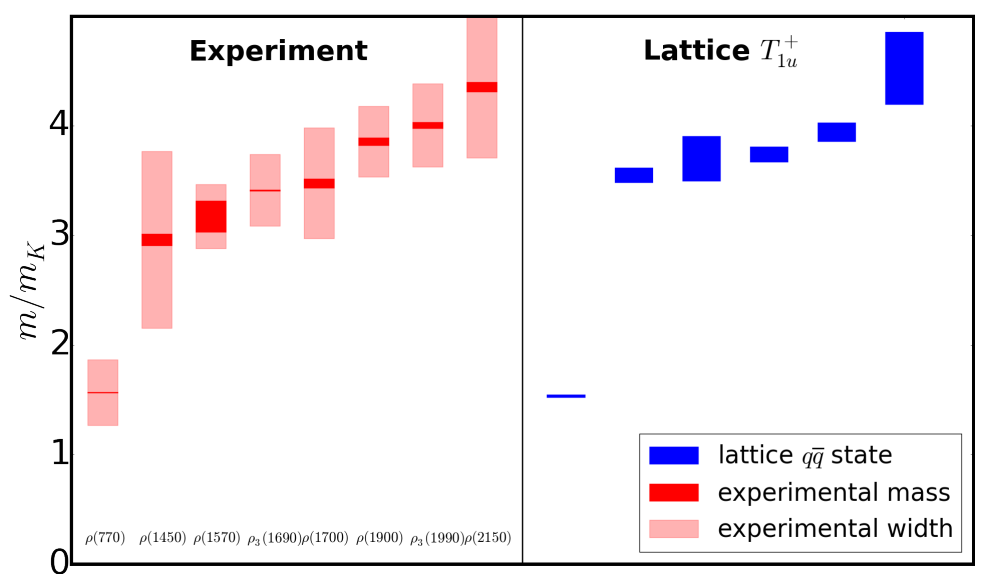

Figure 4: Comparison of the experimental spectrum of resonances with our finite-volume energies corresponding to quark-antiquark excitations. All masses $m$ are shown as ratios over the kaon mass $m_{K}$. In the left hand side, dark red boxes indicate the experimental masses, with the vertical heights showing the uncertainties in the mass measurements. The light red boxes indicate the experimental widths of the resonances. In the right hand side, our masses for the quark-antiquark excitations are shown by dark blue boxes, whose heights indicate statistical uncertainties only. This $T_{1 u}^{+}$channel includes both $\rho$ (spin 1) and $\rho_{3}(\operatorname{spin} 3)$ states.

This work was supported by the U.S. NSF under awards PHY-1306805 and PHY-1318220, and through TeraGrid/XSEDE resources provided by TACC and NICS under grant number TGMCA07S017.

\section{References}

[1] S. Basak, R.G. Edwards, G.T. Fleming, U.M. Heller, C. Morningstar, D. Richards, I. Sato, S. Wallace, Phys. Rev. D 72, 094506 (2005).

[2] S. Basak, R.G. Edwards, G.T. Fleming, K.J. Juge, A. Lichtl, C. Morningstar, D.G. Richards, I. Sato, S.J. Wallace, Phys. Rev. D 76, 074504 (2007).

[3] J. Bulava, R.G. Edwards, E. Engelson, J. Foley, B. Joo, A. Lichtl, H.W. Lin, N. Mathur, C. Morningstar, D.G. Richards, S. Wallace, Phys. Rev. D 79, 034505 (2009).

[4] J. Bulava, R.G. Edwards, E. Engelson, B. Joo, H-W. Lin, C. Morningstar, D.G. Richards, S.J. Wallace, Phys. Rev. D 82, 014507 (2010).

[5] C. Morningstar, J. Bulava, J. Foley, K.J. Juge, D. Lenkner, M. Peardon, C.H. Wong, Phys. Rev. D 83, 114505 (2011)

[6] C. Morningstar, J. Bulava, B. Fahy, J. Foley, Y.C. Jhang, K.J. Juge, D. Lenkner, C.H. Wong, Phys. Rev. D 88, 014511 (2013).

[7] C. Morningstar and M. J. Peardon, Phys. Rev. D 69, 054501 (2004).

[8] M. Peardon, J. Bulava, J. Foley, C. Morningstar, J. Dudek, R. Edwards, B. Joo, H-W. Lin, D. Richards, K.J. Juge, Phys. Rev. D 80, 054506 (2009).

[9] M. A. Clark, A. D. Kennedy, and Z. Sroczynski, Nucl. Phys. B (Proc. Suppl.) 140, 835 (2005).

[10] H.-W. Lin, et al., Phys. Rev. D 79, 034502 (2009). 